\title{
Conhecimento de cirurgiões-dentistas sobre a relação entre disfunção temporomandibular e fatores oclusais
}

\author{
Dentists' knowledge about the relationship between temporomandibular \\ dysfunction and oclusal factors
}

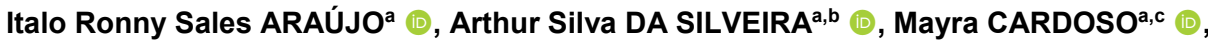 \\ Patricia Nivoloni TANNURE* (1) \\ aUniversidade Veiga de Almeida, Rio de Janeiro, RJ, Brasil \\ bUniversidade do Planalto Central Apparecido dos Santos, Brasília, DF, Brasil \\ 'Universidade do Estado do Rio de Janeiro, Rio de Janeiro, RJ, Brasil
}

\begin{abstract}
Como citar: Araújo IRS, da Silveira AS, Cardoso M, Tannure PN. Conhecimento de cirurgiões-dentistas sobre a relação entre disfunção temporomandibular e fatores oclusais. Rev Odontol UNESP. 2019;48:e20190065. https://doi.org/10.1590/18072577.06519
\end{abstract}

\begin{abstract}
Resumo
Introdução: As evidências científicas atuais têm demonstrado que o tratamento para disfunção temporomandibular deve seguir uma abordagem conservadora e adaptada ao estado psicossocial do paciente. Entretanto, tratamentos visando às alterações oclusais foram utilizados durante anos pelos cirurgiões-dentistas e o abandono destes paradigmas é essencial para o desenvolvimento da Odontologia. Objetivo: Objetivou-se avaliar o conhecimento de cirurgiões-dentistas (CD) sobre a relação entre disfunção temporomandibular (DTM) e fatores oclusais. Material e método: Foi enviado, por e-mail, um questionário previamente publicado para os CD registrados no Conselho Regional de Odontologia do Piauí, Brasil $(\mathrm{n}=2.500)$ com dados sobre o profissional, sua formação e seu conhecimento sobre DTM e fatores oclusais. O consenso da literatura atual, "padrão-ouro", foi comparado com as respostas obtidas. Os dados foram analisados descritivamente e através dos testes qui-quadrado e odds ratio $(\mathrm{p}<0,05)$. Resultado: Quinhentos e cinco CD responderam e 434 compuseram a amostra. A maioria dos participantes era mulher (72,3\%), com 7,2 $( \pm 6,63)$ anos de formados, e trabalhava no setor público-privado $(39,0 \%)$. Os CD foram divididos em dois grupos: GEsp.: especialistas em DTM, Prótese Dentária e/ou Ortodontia (n=234) e GClín.: clínicos gerais e demais especialistas (n=200). GEsp. e GClín. apresentaram respostas similares para a metade das perguntas. Quatro (de seis) perguntas foram respondidas em desacordo com o padrão-ouro: fatores oclusais, interferências em lado de não trabalho, terapia ortodôntica e ajuste oclusal mostraram ser assuntos pouco conhecidos. GEsp. apresentou maior conhecimento com diferença significativa em relação a GClín. acerca da DTM e das interferências oclusais [odds ratio $=2,341(1,305-4,202), p=0,004]$. Conclusão: Pode-se concluir que os CD da amostra estudada apresentaram deficiências acerca do conhecimento da relação entre DTM e fatores oclusais. Especialistas em DTM, Prótese Dentária e/ou Ortodontia não demonstraram um maior nível de conhecimento quando comparados aos demais.
\end{abstract}

Descritores: Oclusão dentária; disfunção temporomandibular; conhecimentos.

\begin{abstract}
Introduction: Current scientific evidence has shown that treatment for temporomandibular disorder should follow a conservative approach that is adapted to the patient's psychosocial status. However, treatments aiming at occlusal changes have been used for years by dentists and the abandonment of these paradigms is essential for the development of dentistry. Objective: The aim of this study was to evaluate the knowledge of dentists on the relationship between temporomandibular disorder (TMD) and occlusal factors. Material and method: A previously published questionnaire was sent to the dentists registered at the Regional Council of Dentistry of Piauí, Brazil $(n=2,500)$ with data about the professional, their training and knowledge about TMD and occlusal factors. The consensus of the current literature, "gold standard", was compared with the answers obtained. The data were analyzed descriptively and through chi-square
\end{abstract}


test and odds ratio ( $\mathrm{p}<0.05)$. Result: Five hundred and five dentists answered and 434 composed the sample. The majority of the participants was women $(72.3 \%)$ with $7.2( \pm 6.63)$ years of education and worked in the public-private sector (39.0\%). The dentists were divided into Group 1 (GEsp.): specialists in TMD, dental prosthesis and/or orthodontics ( $\mathrm{n}=234)$ and Group 2 (GClin.): clinicians and other specialists ( $n=200)$. GEsp. and GClin. presented similar responses for half of the questions. Four (of six) were answered in disagreement with the gold standard: occlusal factors, interference in the balance, orthodontic therapy and occlusal adjustment were little known. GEsp. presented greater knowledge with a significant difference in relation to GClin. regarding TMD and occlusal interferences (odds ratio $=2,341(1,305-4,202)$, $\mathrm{p}=0.004)$. Conclusion: It can be concluded that the dentists presented deficiencies in the knowledge of the relationship between TMD and occlusal factors. Dentists specialists in TMD, dental prosthesis and/or orthodontics did not demonstrate a higher level of knowledge when compared to the others.

Descriptors: Dental occlusion; temporomandibular disorder; knowledge.

\section{INTRODUÇÃO}

A disfunção temporomandibular (DTM) é definida pela American Academy of Orofacial Pain como um grupo de condições musculoesqueléticas e neuromusculares que acometem as articulações temporomandibulares (ATM), os músculos mastigatórios e todos os tecidos associados, e que produzem, como sinais e sintomas, dificuldades na mastigação, na fala e em outras funções orofaciais, podendo, muitas vezes, ser dolorosa ${ }^{1}$. A dor causada pela DTM tem um impacto negativo na qualidade de vida do paciente, prejudicando as atividades do trabalho (73\%), o sono (85\%) e o apetite/ alimentação (82\%) 2 .

De acordo com uma revisão sistemática, a prevalência de DTM observada em adultos com mais de 18 anos e sem diagnóstico prévio da alteração foi de $42,9( \pm 5,8) \%{ }^{3}$. Em estudos realizados no Brasil, podem-se observar altas prevalências de sinais e sintomas de DTM em pré-vestibulandos $(56,4 \%)^{4}$, adolescentes $(33,2 \%)^{5}$, jovens $(43,3 \%)^{6}$ e idosos $(55,1 \%)^{7}$.

A ocorrência de DTM é maior no sexo feminino ${ }^{3,5,6}$, o que pode estar relacionado ao fato de que a proporção de mulheres com patologias ósseas e psicossociais é maior que a proporção de homens afetados ${ }^{8}$. Outra explicação possível seria que, em idade reprodutiva, a prevalência de sinais e sintomas de DTM em mulheres passa a ser maior, pois o estrógeno é o principal contribuinte para a regulação do crescimento e desenvolvimento ósseo, além de influenciar no mecanismo periférico e central da dor'.

Os sinais e sintomas associados à DTM podem variar em sua apresentação e comumente envolvem mais de um componente do sistema estomatognático. Os três principais sinais e sintomas são dor, amplitude de movimento limitada e sons na ATM. A dor é geralmente a queixa principal e pode ser intermitente ou persistente, geralmente de intensidade moderada. Os sintomas mais comuns são dor e sensibilidade à palpação dos músculos pericranianos e da ATM, e muitas vezes eles coexistem ${ }^{10}$.

Atualmente, a DTM é considerada de causa multifatorial e os possíveis fatores envolvidos foram elencados como sendo: trauma direto ou indireto (lesões do tipo chicote); microtrauma (provocado por ações repetitivas, como hábitos parafuncionais - bruxismo, apertamento dentário, etc.); fatores psicossociais (ansiedade, depressão, etc.); fatores fisiopatológicos; fatores sistêmicos (doenças degenerativas, endócrinas, infecciosas, metabólicas, neoplásicas, neurológicas, vasculares e reumatológicas); fatores locais (alteração na viscosidade do líquido sinovial, aumento da pressão intra-articular, estresse oxidativo, etc.), e fatores genéticos (presença de haplótipos associados à sensibilidade dolorosa) ${ }^{11}$.

A relação entre oclusão dentária e DTM tem sido um tema muito discutido na comunidade odontológica. No passado, o estudo da DTM se concentrou em etiologias associadas a fatores únicos, como fármacos, alterações nos músculos ou na oclusão dentária, dando-se ênfase excessiva a esta última. Assim, modificações oclusais irreversíveis foram propostas como 
tratamento, como, por exemplo, o ajuste oclusal, a confecção de próteses ou o tratamento ortodôntico. Entretanto, poucas evidências fortemente suportadas foram apresentadas ${ }^{12}$. Revisões sistemáticas mostraram que não há evidências de uma relação de causa e efeito entre a má oclusão e a disfunção temporomandibular, ou que o ajuste oclusal possa melhorá-la ou evitá-la, sugerindo que os profissionais sejam desencorajados a continuar com a realização de procedimentos com base nos conceitos antigos que relacionam DTM aos desarranjos oclusais ${ }^{13,14}$

O consenso atual é de que as modalidades de tratamento para DTM devem ser conservadoras, reversíveis e não invasivas, sempre que possível, e devem ser adaptadas ao estado psicossocial do paciente. Muitos desses tratamentos estão disponíveis e podem ser administrados em um ambiente multidisciplinar. Os elementos-chave de qualquer plano de manejo conservador são o autocuidado, a medicação e a fisioterapia. A acupuntura geralmente é útil, assim como a terapia com placa oclusal e terapia manual. Também pode ser utilizada, de acordo com o caso, a injeção de anestesia local, artrocentese e artroscopia. Em menos de 5\% dos casos, há necessidade de cirurgia 15

Devido às muitas controvérsias que o tema abordado traz, o conhecimento de uma odontologia baseada em evidências é necessário para que o profissional seja bem orientado na sua prática clínica. Sob essas circunstâncias, a probabilidade de erro pode ser reduzida, garantindo a melhor tomada de decisão para o tratamento a ser instituído. Deste modo, o objetivo desse estudo é avaliar o nível de conhecimento de cirurgiões-dentistas sobre a relação entre DTM e fatores oclusais.

\section{MATERIAL E MÉTODO}

O estudo foi aprovado pelo Comitê de Ética em Pesquisa da Universidade Veiga de Almeida Rio de Janeiro (parecer n.o 2.593.320), respeitando os critérios da Resolução n. $466 / 2012$ do Conselho Nacional de Saúde.

A população pesquisada constituiu-se de 2.500 cirurgiões-dentistas registrados no Conselho Regional de Odontologia (CRO) do Estado do Piauí, no período da coleta de dados (junho de 2018). 0 questionário foi elaborado a partir da plataforma de formulários SurveyMonkey (pt.surveymonkey.com) e, em seguida, foi enviado por e-mail a todos os cirurgiões-dentistas registrados. Foi utilizado um questionário previamente publicado por Assis et al. ${ }^{16}$ contendo perguntas relevantes acerca da relação entre DTM e fatores oclusais.

0 questionário era dividido em três partes. A primeira era constituída de questões gerais, como idade, sexo, tempo e local de graduação. A segunda parte apresentava perguntas sobre dados específicos, como a formação na graduação, pós-graduação, fontes de atualização e conduta frente ao paciente portador de DTM. A terceira e última parte continha perguntas para avaliar o conhecimento sobre a relação entre fatores oclusais e DTM. Nesta etapa, os participantes poderiam responder de 0 a 2, em que 0 (zero) significava "não concordo"; 1 significava "não sei responder", e 2 significava "concordo". Somente foram incluídos os questionários que continham todas as perguntas respondidas, oferecendo informações necessárias para atender aos objetivos do estudo.

Para análise e comparação, os grupos foram divididos em: GEsp (grupo de especialistas em DTM/dor orofacial, Ortodontia e/ou Prótese Dentária) e GClín (grupo de clínicos gerais ou outras especialidades). Utilizou-se dos valores consensuais sobre a relação entre DTM e oclusão já publicados, considerando que o "padrão-ouro" das respostas é baseado nas últimas evidências científicas que regem o tema ${ }^{16}$, como mostra o Quadro 1.

Para a análise estatística, foi utilizado o teste do qui-quadrado em um nível de significância de 5\%, através do programa estatístico IBM SPSS (Statistics Statistical Package for Social Sciences), versão 25.0 (SPSS Inc, Chicago, Illinois, EUA). 
Quadro 1. Valores de consenso ("padrão-ouro") para cada uma das afirmações ao avaliar o conhecimento sobre a relação entre oclusão e disfunções temporomandibulares

\begin{tabular}{|l|c|}
\hline \multicolumn{1}{|c|}{ Afirmação } & Resposta ideal \\
\hline Os fatores oclusais dos pacientes podem ser a causa principal da DTM. & Não concordo \\
\hline $\begin{array}{l}\text { Interferências no lado balanceio são frequentemente relacionadas com } \\
\text { a DTM. }\end{array}$ & Não concordo \\
\hline Bruxismo noturno é causado por interferências oclusais. & Não concordo \\
\hline $\begin{array}{l}\text { Hábitos parafuncionais orais são muitas vezes significativos no } \\
\text { desenvolvimento da DTM }\end{array}$ & Concordo \\
\hline $\begin{array}{l}\text { A terapia ortodôntica é o melhor tratamento para resolver DTM com } \\
\text { má oclusão. }\end{array}$ & Não concordo \\
\hline O ajuste oclusal é um tratamento útil na prevenção da DTM & Não concordo \\
\hline
\end{tabular}

Fonte: Extraído de Assis et al. ${ }^{16}$.

\section{RESULTADO}

Quinhentos e cinco profissionais responderam ao questionário, dos quais 501 (99,21\%) aceitaram participar da pesquisa. 0 número dos que concluíram a pesquisa e foram considerados elegíveis foi de 434 .

Um total de $314(72,35 \%)$ da amostra elegível foi constituído por participantes do sexo feminino. A média de tempo de formado foi de $7,2( \pm 6,63)$ anos. A maioria dos participantes obteve conhecimentos durante a graduação sobre oclusão e DTM. Aproximadamente 55\% destes responderam que esses conhecimentos foram ministrados em uma mesma disciplina, seguidos daqueles que adquiriram em disciplinas diferentes (38\%). Quando questionados sobre a área de atuação, 31\% relataram atuação em consultório particular e aproximadamente $29 \%$ em serviço público, enquanto que $39 \%$ atuavam em ambas as modalidades.

Um total de 51 participantes não relatou cursos de pós-graduação. Aqueles que detinham algum curso na área de DTM/dor orofacial eram seis. Profissionais que tinham cursos de Mestrado/Doutorado eram 35, enquanto que a grande maioria (263) apresentava especialidades que não apareciam nas opções. No entanto, essas especialidades foram mencionadas, a saber: Ortodontia (218); Prótese Dentária (16); Endodontia (15); Implantodontia (8); Cirurgia Bucomaxilofacial (7); Odontopediatria (7); Saúde da Família/Coletiva/Pública (6); Periodontia (4); Docência em Ensino Superior (2); Dentística (2); Imaginologia/ Radiologia (2); Harmonização Orofacial (1); Oncologia (1); Pacientes com Necessidades Especiais (1). Quando as perguntas abordavam a conduta no atendimento aos pacientes com DTM, 181 (41\%) responderam que tratavam e 277 (63\%) encaminhavam os pacientes.

No tocante à terceira parte, que incluía dados sobre os conhecimentos de oclusão e DTM, as afirmações e a porcentagem dos respondentes estão detalhadas na Tabela 1. Na Tabela 2, estão relatados os dados e as análises estatísticas referentes às respostas do questionário em comparação com as respostas consensuais (padrão-ouro).

Tabela 1. Dados obtidos a partir das respostas do questionário aplicado 


\begin{tabular}{|c|c|c|c|c|c|c|}
\hline \multirow{3}{*}{ Afirmações } & \multicolumn{6}{|c|}{ Respostas: GEsp. $(\mathrm{n}=234)$, GClín. $(\mathrm{n}=200)$} \\
\hline & \multicolumn{2}{|c|}{ Não concordaram } & \multicolumn{2}{|c|}{$\begin{array}{c}\text { Não souberam } \\
\text { responder }\end{array}$} & \multicolumn{2}{|c|}{ Concordaram } \\
\hline & GEsp.(\%) & GClín.(\%) & GEsp.(\%) & GClín.(\%) & GEsp. $(\%)$ & GClín.(\%) \\
\hline $\begin{array}{l}\text { 1-Os fatores oclusais dos } \\
\text { pacientes podem ser a causa } \\
\text { principal da DTM. }\end{array}$ & $63(27)$ & $78(39)$ & $6(3)$ & $16(8)$ & $165(71)$ & $106(53)$ \\
\hline $\begin{array}{l}\text { 2- Interferências no lado } \\
\text { balanceio são frequentemente } \\
\text { relacionadas com a DTM. }\end{array}$ & $44(19)$ & $18(9)$ & $45(19)$ & $103(52)$ & $145(62)$ & $79(40)$ \\
\hline $\begin{array}{l}\text { 3-Bruxismo noturno é causado } \\
\text { por interferências oclusais. }\end{array}$ & $168(72)$ & $161(81)$ & $25(11)$ & $8(4)$ & $41(18)$ & $31(16)$ \\
\hline $\begin{array}{l}\text { 4-Hábitos parafuncionais orais } \\
\text { são muitas vezes significativos no } \\
\text { desenvolvimento da DTM. }\end{array}$ & $14(6)$ & $8(4)$ & $15(6)$ & $6(3)$ & $205(88)$ & $186(93)$ \\
\hline $\begin{array}{l}\text { 5-A terapia ortodôntica é o } \\
\text { melhor tratamento para resolver } \\
\text { DTM com má oclusão. }\end{array}$ & $112(48)$ & $90(45)$ & $4(2)$ & $65(33)$ & $118(50)$ & $45(23)$ \\
\hline $\begin{array}{l}\text { 6-0 ajuste oclusal é um } \\
\text { tratamento útil na prevenção da } \\
\text { DTM. }\end{array}$ & $48(21)$ & $50(25)$ & $28(12)$ & $12(6)$ & $158(68)$ & 138 (69) \\
\hline
\end{tabular}

GEsp.: Grupo de especialistas em DTM/dor orofacial, Ortodontia e/ou Prótese Dentária; GClín.: Grupo de clínicos gerais (não especialistas) ou especialistas em outras áreas.

Tabela 2. Análise estatística com o teste do qui-quadrado e estimativa de risco (odds ratio) das respostas dos grupos

\begin{tabular}{|c|c|c|c|c|c|}
\hline \multirow[t]{2}{*}{ Afirmações } & \multirow{2}{*}{$\begin{array}{l}\text { Relação com o } \\
\text { padrão-ouro }\end{array}$} & $\begin{array}{c}\text { GEsp } \\
(\%)\end{array}$ & $\begin{array}{c}\text { GClín } \\
(\%)\end{array}$ & $\begin{array}{c}\text { Estimativa de } \\
\text { risco }\end{array}$ & \multirow{2}{*}{$\begin{array}{l}\text { Valor de } \\
\text { p }\end{array}$} \\
\hline & & $(n=234)$ & $(n=200)$ & (IC 95\%) & \\
\hline 1 - Os fatores oclusais dos & Em concordância & $63(27)$ & $78(39)$ & $0,5760,576$ & \multirow[b]{2}{*}{$0,007^{*}$} \\
\hline $\begin{array}{l}\text { pacientes podem ser a causa } \\
\text { principal da DTM. }\end{array}$ & Em discordância & $171(73)$ & $122(61)$ & $(0,384-0,864)$ & \\
\hline 2 - Interferências no lado & Em concordância & $44(19)$ & $18(9)$ & $2,3412,341$ & \multirow[b]{2}{*}{$0,004^{*}$} \\
\hline $\begin{array}{l}\text { balanceio são frequentemente } \\
\text { relacionadas com a DTM. }\end{array}$ & Em discordância & $190(81)$ & $182(91)$ & $(1,305-4,202)$ & \\
\hline \multirow{2}{*}{$\begin{array}{l}3 \text { - Bruxismo noturno é causado } \\
\text { por interferências oclusais. }\end{array}$} & Em concordância & $168(72)$ & $161(81)$ & $0,6170,617$ & \multirow{2}{*}{$0,035^{*}$} \\
\hline & Em discordância & $66(28)$ & $39(20)$ & $(0,393-0,968)$ & \\
\hline \multirow{2}{*}{$\begin{array}{l}4 \text { - Hábitos parafuncionais orais } \\
\text { são muitas vezes significativos no } \\
\text { desenvolvimento da DTM. }\end{array}$} & Em concordância & $205(88)$ & $186(93)$ & 0,532 & \multirow[b]{2}{*}{$0,061 \mathrm{NS}$} \\
\hline & Em discordância & $29(12)$ & $14(7)$ & $(0,273-1,038)$ & \\
\hline \multirow{2}{*}{$\begin{array}{l}5 \text { - A terapia ortodôntica é o } \\
\text { melhor tratamento para resolver } \\
\text { DTM com má oclusão. }\end{array}$} & Em concordância & $112(48)$ & $90(45)$ & 1,122 & \multirow[b]{2}{*}{$0,551 \mathrm{NS}$} \\
\hline & Em discordância & $122(52)$ & $110(55)$ & $(0,768-1,639)$ & \\
\hline \multirow{2}{*}{$\begin{array}{l}6 \text { - } 0 \text { ajuste oclusal é um } \\
\text { tratamento útil na prevenção da } \\
\text { DTM. }\end{array}$} & Em concordância & $48(21)$ & $50(25)$ & 0,774 & \multirow[b]{2}{*}{$0,265 \mathrm{NS}$} \\
\hline & Em discordância & $186(79)$ & $150(75)$ & $(0,493-1,215)$ & \\
\hline
\end{tabular}

IC: Intervalo de confiança; NS: Não-significativo; ${ }^{*}$ Estatisticamente significativo através do teste do qui-quadrado.

\section{DISCUSSÃO}

Este estudo avaliou o nível de conhecimento de cirurgiões-dentistas do estado do Piauí sobre a relação entre DTM e fatores oclusais. Observou-se que os profissionais apresentaram deficiências acerca do conhecimento da relação entre DTM e fatores oclusais. Especialistas em 
DTM, Prótese Dentária e/ou Ortodontia não demonstraram um maior nível de conhecimento quando comparados aos demais.

Na presente pesquisa, o número de entrevistados do sexo feminino em relação ao masculino foi maior, na proporção de 2:1. Dados regionais corroboram esses resultados ${ }^{17}$. Essa diferença, no Brasil, é fruto do processo de modernização e de mudança cultural do país, sendo cada vez mais intensa a associação entre a escolaridade e a participação das mulheres no mercado de trabalho ${ }^{18}$.

Há de se ponderar um número baixo de especialistas em DTM/dor orofacial (seis) observado durante a pesquisa (número de especialistas registrados no CRO-PI). Não significa, entretanto, que $100 \%$ dos especialistas dessa região responderam ao questionário. Isto pode ser explicado pelo fato de que alguns profissionais não registraram suas especialidades. 0 respondente pode também ter realizado cursos de atualização/aperfeiçoamento na área, o que não foi considerado como especialização. No Brasil, o número de profissionais especialistas inscritos no Conselho Federal é de 1.303 , o que representa $1,1 \%$ do total de profissionais registrados. Uma das especialidades mais mencionadas nesse estudo foi a Ortodontia (218), o que vai ao encontro das informações disponibilizadas pelo CFO, que a menciona como a especialidade odontológica mais frequente no Brasil ( $23 \%$ dos registrados).

Durante a graduação, muitos cursos de Odontologia ministram aulas sobre DTM e/ou oclusão, pelo que se presume que o egresso seja conhecedor destes temas. Nesta pesquisa, observou-se que $92 \%$ dos participantes relataram ter estudado estes temas na mesma disciplina ou de forma isolada. Até o presente momento, o tema DTM/dor orofacial não é tópico obrigatório do conteúdo programático das instituições de ensino de Odontologia. Esse fato, quando negligenciado, pode levar a uma falta de preparo do cirurgião-dentista para diagnosticar, tratar ou orientar os portadores desses distúrbios ${ }^{11}$.

$\mathrm{Na}$ interação entre dois fenômenos, quanto mais forte essa associação, maior será a possibilidade de se tratar de uma relação causal. Em outras palavras, certa característica oclusal deve estar mais presente em pacientes com DTM do que em indivíduos saudáveis, e mais frequentemente ausente em indivíduos saudáveis do que em pacientes com DTM. Sabe-se que a demonstração de uma associação é apenas o primeiro critério a se considerar como base para uma possível relação de causa e efeito entre as duas condições. Quando confirmada pela maioria das pesquisas, tal associação pode ser considerada consistente.

Fundamentado nisso, várias revisões sistemáticas buscaram resposta sobre a associação entre má oclusão e DTM. Essas revisões chegaram a uma conclusão em comum: há ausência de evidência de que o ajuste oclusal trata ou previne a DTM16,19,20. Contudo, é importante ter em mente que existe diferença entre ausência de evidência e evidência de ausência. Pode não haver evidência de um efeito porque existem poucos dados sobre sua efetividade. 0 ajuste oclusal, devido a isso, é contraindicado no tratamento ou prevenção da DTM ${ }^{19}$. Como demonstrado na Tabela 1, observou-se que $71 \%$ dos especialistas concordaram com a afirmação de que os fatores oclusais podem ser a causa principal da DTM e 39\% dos não especialistas concordaram com a afirmação, mesmo com a literatura sendo bem enfática preconizando o contrário.

Quando questionados se interferências no lado balanceio são frequentemente relacionadas com a DTM, a maioria dos participantes respondeu contrário às evidências. No entanto, o número de entrevistados que não souberam responder foi elevado (148; 34\%). Embora a afirmação de que interferências no lado de balanceio (não trabalho) se relacionem com disfunção temporomandibular possa parecer um tema menos conhecido, a literatura não suporta essa relação, mostrando estudos em que alguns pacientes desenvolvem os sinais e sintomas de DTM mesmo quando não há interferência e que alguns indivíduos com interferência do lado do balanceio também não apresentam esse distúrbio ${ }^{21}$.

Estudiosos argumentaram que nem todo bruxista tem interferências oclusais e nem todo indivíduo com tais interferências é bruxista. Esses autores também afirmaram que existem 
evidências convincentes de que o bruxismo é principalmente mediado centralmente, e não perifericamente, isto é, não causado por fatores anatômicos como certas características de oclusão dentária e articulação ${ }^{22}$. Quanto à etiologia, o bruxismo pode ser considerado multifatorial e há fortes evidências de que o papel das características oclusais e de outros fatores morfológicos é pequeno, se é que está presente ${ }^{23}$. Portanto, não há evidências de que contatos prematuros ou interferências oclusais estariam relacionados com a etiologia do bruxismo ${ }^{23}$. A maioria dos participantes dos dois grupos (72\% para o G1 e 81\% para o G2) respondeu em concordância com o embasamento científico.

De acordo com o resultado da pesquisa, também foi observado que $88 \%$ do grupo de especialistas e que a grande maioria do grupo clínico (93\%) concordaram que hábitos parafuncionais orais são muitas vezes significativos no desenvolvimento da DTM. Hábitos parafuncionais geralmente estão relacionados ao bruxismo (ranger ou apertar), mastigação excessiva de produtos, como goma de mascar, mordedura labial ou sucção não nutritiva ${ }^{24}$. Esses comportamentos afetam o funcionamento normal das estruturas orais e músculos associados. Corroborando os resultados desta pesquisa, a literatura é bem vasta quando diz respeito ao favorecimento que estes hábitos podem causar na incidência de DTM²4-26.

A relação entre Ortodontia e DTM é complexa e controversa. Embora haja um conceito empírico de que a intervenção ortodôntica possa causar DTM, ao analisar as últimas evidências, observou-se que o tratamento ortodôntico não predispõe a esses distúrbios. Além disso, associações entre tipos específicos de más oclusões e desenvolvimento de sinais e sintomas de DTM não puderam ser verificadas e parece não haver evidências de uma relação de causa-efeito direta ou óbvia entre o tratamento ortodôntico e a DTM14,19. Consequentemente, não há evidências de que a Ortodontia possa corrigir ou prevenir a DTM, embora mais estudos longitudinais sejam necessários para verificar qualquer possível inter-relação. Os participantes desta pesquisa, de ambos os grupos, opinaram de forma peculiar em comparação às demais afirmações. Pode-se observar um equilíbrio nestas respostas, o que demonstra que ainda pairam dúvidas acerca da conduta frente ao paciente com esses distúrbios.

Quando questionados se o ajuste oclusal é um tratamento útil na prevenção da DTM, a grande maioria do grupo de especialistas e dos clínicos concordou com a afirmação. Não obstante, Manfredini et al. ${ }^{14}$ são bem incisivos ao afirmar que se deve colocar um ponto final no pensamento que diz que a variabilidade nas características interindividuais da oclusão dentária é considerada um sinal patológico no campo da DTM, aconselhando os clínicos a abandonar este paradigma antiquado.

De acordo com os resultados da Tabela 2, notam-se diferenças significativas entre os grupos para as afirmações 01,02 e $03(\mathrm{p}<0,05)$, através do teste do qui-quadrado. Também se pode inferir que ser especialista reduziu em cerca de $42 \%$ a chance de responder de acordo com o padrão-ouro, quando analisada a afirmação "Os fatores oclusais dos pacientes podem ser a causa principal da DTM". Ser especialista também reduziu em cerca de $38 \%$ a chance de responder em concordância à afirmação "Bruxismo noturno é causado por interferências oclusais", enquanto que ser especialista aumentou em 2,3 vezes a chance de responder de acordo com os padrões-ouro à afirmação "Interferências no lado balanceio são frequentemente relacionadas com a DTM".

Vale relatar aqui uma limitação deste estudo: um elevado número de formulários enviados por e-mail poderia ter sido direcionado para caixa de spam ou lixeira dos correios eletrônicos dos entrevistados, impossibilitando que esta pesquisa tenha sido respondida por mais sujeitos. Entretanto, o baixo custo, a facilidade e a rapidez para acessar um grande número de respondentes faz com que o envio de um questionário online seja uma ferramenta interessante de pesquisa nos dias atuais. 


\section{CONCLUSÃO}

Pode-se concluir que a amostra estudada de cirurgiões-dentistas apresentou deficiências acerca do conhecimento da relação entre fatores oclusais e disfunção temporomandibular, acreditando haver uma relação mais forte do que a literatura atual demonstra. Especialistas em DTM, Prótese Dentária e/ou Ortodontia não apresentaram um maior nível de conhecimento quando comparados aos demais.

\section{REFERÊNCIAS}

1. Greene CS. Managing patients with temporomandibular disorders: a new "standard of care". Am J Orthod Dentofacial Orthop. 2010 Jul;138(1):3-4. http://dx.doi.org/10.1016/j.ajodo.2010.04.015. PMid:20620818.

2. Moreno BGD, Maluf SA, Marques AP, Crivello-Júnior O. Avaliação clínica e da qualidade de vida de indivíduos com disfunção temporomandibular. Rev Bras Fisioter. 2009 Jun;13(3):210-4. http://dx.doi.org/10.1590/S1413-35552009005000028.

3. Bueno CH, Pereira DD, Pattussi MP, Grossi PK, Grossi ML. Gender differences in temporomandibular disorders in adult populational studies: a systematic review and meta-analysis. J Oral Rehabil. 2018 Sep;45(9):720-9. http://dx.doi.org/10.1111/joor.12661. PMid:29851110.

4. Paulino MR, Moreira VG, Lemos GA, Silva PLP, Bonan PRF, Batista AUD. Prevalence of signs and symptoms of temporomandibular disorders in college preparatory students: associations with emotional factors, parafunctional habits, and impact on quality of life. Cien Saude Colet. 2018 Jan;23(1):173-86. http://dx.doi.org/10.1590/1413-81232018231.18952015. PMid:29267822.

5. Pereira LJ, Pereira-Cenci T, Pereira SM, Cury AA, Ambrosano GM, Pereira AC, et al. Psychological factors and the incidence of temporomandibular disorders in early adolescence. Braz Oral Res. 2009 AprJun;23(2):155-60. http://dx.doi.org/10.1590/S1806-83242009000200011. PMid:19684950.

6. Progiante PS, Pattussi MP, Lawrence HP, Goya S, Grossi PK, Grossi ML. Prevalence of temporomandibular disorders in an adult brazilian community population using the research diagnostic criteria (axes I and II) for temporomandibular disorders (the Maringá study). Int J Prosthodont. 2015 Nov-Dec;28(6):600-9. http://dx.doi.org/10.11607/ijp.4026. PMid:26523719.

7. - Czernaik CM, Muniz FWMG, Colussi PRG, Rösing CK, Colussi, EL. Association between temporomandibular disorder symptoms and demographic, dental and behavioral factors in the elderly: a population-based cross-sectional study. Br J P. 2018 Jul;1(3):223-30.

8. Kostrzewa-Janicka J, Pietrzak B, Jurkowski P, Wielgos M, Binkowska M, Mierzwinska-Nastalska E. Effects of oral contraceptives on the treatment for internal derangements in temporomandibular joints in women. Neuroendocrinol Lett. 2013;34(6):566-72. PMid:24378447.

9. Kim TY, Shin JS, Lee J, Lee YJ, Kim MR, Ahn YJ, et al. Gender difference in associations between chronic temporomandibular disorders and general quality of life in Koreans: a cross-sectional study. PLoS One. 2015 Dec;10(12):e0145002. http://dx.doi.org/10.1371/journal.pone.0145002. PMid:26673219.

10. Schiffman EL, Truelove EL, Ohrbach R, Anderson GC, John MT, List T, et al. The research diagnostic criteria for temporomandibular disorders. I: overview and methodology for assessment of validity. J Orofac Pain. 2010;24(1):7-24. PMid:20213028.

11. Carrara SV, Conti PCR, Barbosa JS. Termo do $1^{\circ}$ consenso em disfunção temporomandibular e dor orofacial. Dental Press J Orthod. 2010 Jun;15(3):114-20. http://dx.doi.org/10.1590/S217694512010000300014.

12. Ohrbach R, Dworkin SF. The evolution of TMD diagnosis: past, present, future. J Dent Res. 2016 Sep;95(10):1093-101. http://dx.doi.org/10.1177/0022034516653922. PMid:27313164. 
13. Rinchuse DJ, Rinchuse DJ, Kandasamy S. Evidence-based versus experience-based views on occlusion and TMD. Am J Orthod Dentofacial Orthop. 2005 Feb;127(2):249-54. http://dx.doi.org/10.1016/j.ajodo.2004.02.012. PMid:15750546.

14. Manfredini D, Lombardo L, Siciliani G. Temporomandibular disorders and dental occlusion. A systematic review of association studies: end of an era? J Oral Rehabil. 2017 Nov;44(11):908-23. http://dx.doi.org/10.1111/joor.12531. PMid:28600812.

15. Goddard G, Mauro G. Temporomandibular disorders, a review of current diagnosis and treatment. Dent Cadmos. 2018;86(5):364-75. http://dx.doi.org/10.19256/d.cadmos.05.2018.04.

16. Assis JFC, Silva PLP, Lima JAS, Forte FDS, Batista AUD. The knowledge level of dental surgeons regarding the relationship between occlusal factors and Temporomandibular Disorders (TMD). Rev Odontol UNESP. 2015 Dec;44(6):360-7. http://dx.doi.org/10.1590/1807-2577.11615.

17. Moura MS, Ferro FE, Cunha NL, Nétto OB, Lima MD, Moura LF. Saúde bucal na Estratégia de Saúde da Família em um colegiado gestor regional do estado do Piauí. Cien Saude Colet. 2013 Fev;18(2):471-80 http://dx.doi.org/10.1590/S1413-81232013000200018. PMid:23358772.

18. Bruschini MCA. Trabalho e gênero no Brasil nos últimos dez anos. Cadernos Pesquisa. 2007;37(132):537-72. http://dx.doi.org/10.1590/S0100-15742007000300003.

19. Manfredini D, Stellini E, Gracco A, Lombardo L, Nardini LG, Siciliani G. Orthodontics is temporomandibular disorder-neutral. Angle Orthod. 2016 Jul;86(4):649-54. http://dx.doi.org/10.2319/051015-318.1. PMid:26512757.

20. Kanter RJAM, Battistuzzi PGFCM, Truin G-J. Temporomandibular disorders: "occlusion" matters! Pain Res Manag. 2018 May;2018:8746858. http://dx.doi.org/10.1155/2018/8746858. PMid:29861806.

21. Türp JC, Schindler H. The dental occlusion as a suspected cause for TMDs: epidemiological and etiological considerations. J Oral Rehabil. 2012 Jul;39(7):502-12. http://dx.doi.org/10.1111/j.13652842.2012.02304.x. PMid:22486535.

22. Lauriti L, Motta LJ, Godoy CH, Biasotto-Gonzalez DA, Politti F, Mesquita-Ferrari RA, et al. Influence of temporomandibular disorder on temporal and masseter muscles and occlusal contacts in adolescents: an electromyographic study. BMC Musculoskelet Disord. 2014 Apr;15(1):123. http://dx.doi.org/10.1186/1471-2474-15-123. PMid:24721559.

23. Lobbezoo F, Naeije M. Bruxism is mainly regulated centrally, not peripherally. J Oral Rehabil. 2001 Dec;28(12):1085-91. http://dx.doi.org/10.1046/j.1365-2842.2001.00839.x. PMid:11874505.

24. Kato T, Thie NM, Huynh N, Miyawaki S, Lavigne GJ. Topical review: sleep bruxism and the role of peripheral sensory influences. J Orofac Pain. 2003;17(3):191-213. PMid:14520766.

25. Fale H, Hnamte L, Deolia S, Pasad S, Kohale S, Sen S. Association between parafunctional habit and sign and symptoms of temporomandibular dysfunction. J Dent Res Rev. 2018;5(1):17-21.

http://dx.doi.org/10.4103/jdrr.jdrr_1_18.

26. Bruguiere F, Sciote JJ, Roland-Billecart T, Raoul G, Machuron F, Ferri J, et al. Pre-operative parafunctional or dysfunctional oral habits are associated with the temporomandibular disorders after orthognathic surgery: an observational cohort study. J Oral Rehabil. 2019 Apr;46(4):321-9. http://dx.doi.org/10.1111/joor.12749. PMid:30472807.

\section{CONFLITOS DE INTERESSE}

Os autores declaram não haver conflitos de interesse. 


\section{*AUTOR PARA CORRESPONDÊNCIA}

Patricia Nivoloni Tannure - Universidade Veiga de Almeida, Rua Ibituruna, 108, Maracanã 20271-020 Rio de Janeiro - RJ, Brasil, e-mail: pntannure@gmail.com

Recebido: Junho 25, 2019

Aprovado: Setembro 30, 2019 